\title{
versants
}

\section{L'Onegin di Giovanni Giudici. Dal laboratorio poetico}

\author{
Sara Cerneaz \\ Università di Zurigo / Università degli Studi di Udine
}

\begin{abstract}
Per la prima volta dagli anni Settanta, Joanna Spendel offre un racconto della traduzione dell'Onegin di Puškin che a lungo l'ha legata a Giovanni Giudici. Ne emerge un ritratto dell'intimo sodalizio con il poeta, ma soprattutto, grazie a materiale inedito, la testimonianza fino ad ora sconosciuta dell'officina di traduzione. Un'occasione unica per entrare nel laboratorio poetico dell'opera che ha accompagnato Giudici per quasi quarant'anni.
\end{abstract}

Keywords: Giovanni Giudici, Joanna Spendel, Onegin, poesia, traduzione.

Giovanni Giudici (1924-20II), tra i più importanti poeti del secondo '90o italiano, ha lasciato diverse testimonianze di come lavorava ai suoi versi. La più completa, quella che rivela un rapporto privilegiato con la scrittura a macchina, è data in un celebre saggio, intitolato Le macchine del poeta:

La genesi del testo eccola qui: parto da un appunto scritto a mano, su un taccuino, e trasferito poi su un'agenda da tavolo che riempio via via senza tener conto della numerazione dei giorni, metto un foglio in macchina e parto con un primo verso puntando al compimento di una prima strofa; ma può succedere (e quasi sempre) che già al secondo verso io mi accorga di battere una pista sbagliata; e allora, subito, via il foglio, inserisco un foglio nuovo, ricomincio; e così di questo passo, finché la poesia non raggiunga un assetto sufficientemente concluso, da prima stesura... A volte quattro o cinque fogli mi bastano, ma in certi casi me ne occorrono decine e decine [...] Ma, abbiate pazienza, non è finita: nessuna prima stesura è, infatti, definitiva, dopo di essa il "dossier del poema" continua a crescere; correggo a mano un verso, una parola, e subito una nuova "bella copia" si rende necessaria; o, per un semplice gusto della forma, decido di usare un diverso carattere dattilografico e ancora ricopio e, nel ricopiare, è piuttosto improbabile che non si affacci la tentazione di ulteriori varianti. ${ }^{1}$

Anche per l'opera di traduzione, a cui Giudici si dedica ampiamente, il poeta riferisce un lavoro pressoché diretto alla macchina da scrivere. Della traduzione di Robert Frost racconta: ${ }^{2}$

I Giovanni Giudici, Le macchine del poeta, in Id., La dama non cercata. Poetica e letteratura (I9681984), Milano, Mondadori, I985, pp. I49-I54, pp. 152-153.

2 Robert Frost, Conoscenza della notte e altre poesie, Torino, Einaudi, 1965. 
Testo originale a sinistra, macchina per scrivere davanti a me e dizionario Webster sulla destra, traducevo in prima stesura quasi come se traducessi in prosa, badando anzitutto ai significati letterali, di grado zero. ${ }^{3}$

La «temeraria impresa» ${ }^{4}$ di traduzione dell'Evgenij Onegin di Puškin, romanzo in versi capolavoro della letteratura russa dell'Ottocento a cui il poeta si dedica instancabilmente dagli anni '70, è invece descritta così:

Il mio primo strumento di traduttore, quando verso il 1970 decisi di dedicarmi con un quasi preciso impegno all'impresa, fu naturalmente il vocabolario; ma, accanto al vocabolario, tenuto presente che tra le componenti della lingua poetica il momento ritmico-fonico non è meno importante del momento sintattico-lessicale, di grandissimo aiuto mi furono i dischi sui quali un grande attore russo, Vsevolod Aksënov, aveva inciso la sua mirabile dizione. ${ }^{5}$

Ma è importante soffermarsi anche sul ruolo riconosciuto alla slavista Joanna Spendel. Nella prima pubblicazione parziale dell'opera, sull'«Almanacco dello Specchio» I972, il suo nome compare in qualità di curatrice delle note. ${ }^{6}$ Dall'edizione completa del 1975 Spendel firma anche l'introduzione ed è ricordata nella nota del traduttore: «a lei - scrive Giudici - sono poi grato per l'aiuto che ha voluto darmi nel corso del lavoro e specialmente nella revisione finale». ${ }^{7}$ Un ricordo che diventa più sentito nell'edizione I983: «Prezioso, per non dire indispensabile, mi è stato in tale revisione il contributo di Giovanna Spendel: a lei va il mio grazie». ${ }^{8}$ Dall'edizione Fogola del I990, ${ }^{9}$ come poi nella collana "gli elefanti" del 1999, ${ }^{\text {I0 }}$ Spendel non viene in-

3 Giovanni Giudici, Da un'officina di traduzioni, in Id., Per forza e per amore, Milano, Garzanti, I996, pp. 20-33, p. 27.

4 Giovanni Giudici, Per passione e su commissione, in Id., Addio, proibito piangere e altri versi tradotti (1955-I980), Torino, Einaudi, I982, pp. V-XI, p. X.

5 Giovanni Giudici, Per un Onieghin italiano, in Giovanni Giudici, Eugenio Onieghin di Aleksandr S. Puškin in versi italiani, prefazione di Gianfranco Folena, Milano, Garzanti, 1999, pp. I9I-I99, p. 197 .

6 Aleksandr Sergeevič Puškin, Evgenij Onegin, dedica e primo capitolo, introduzione e traduzione di Giovanni Giudici, brevi note a cura di Joanna Spendel, in «Almanacco dello Specchio», I972, I, a cura di Marco Forti e con la collaborazione di Giuseppe Pontiggia, pp. I4-45.

7 Aleksandr Sergeevič Puškin, Evgenij Onegin (Eugenio Onieghin), traduzione in versi italiani di Giovanni Giudici, Milano, Garzanti, 1975, p. XXV.

8 Giovanni Giudici, Eugenio Onieghin di Puškin in versi italiani, prefazione di Gianfranco Folena, Milano, Garzanti, 1983, p. XVII.

9 Aleksandr Sergeevič Puškin, Eugenio Onieghin, traduzione in versi italiani di Giovanni Giudici, Torino, Fogola, 1990.

Io Giovanni Giudici, Eugenio Onieghin di Aleksandr S. Puškin in versi italiani, prefazione di Gianfranco Folena, Milano, Garzanti, 1999. Per la descrizione completa delle diverse edizioni dell'opera rimando a: Sara Cerneaz, L'Onegin di Giudici: un'analisi metrico-variantistica, tesi di dottorato in co-tutela tra Universität Zürich e Università degli Studi di Udine, approvata dalla 
vece più nominata. Tale sviluppo è certamente connesso con le interessanti vicende editoriali e peritestuali dell'opera. Se Giudici già nell'edizione del I975 parla della collocazione del suo lavoro «nell'ambito della letteratura italiana» e di un "momento della sua ricerca poetica», " tuttavia il suo nome si legge solo nel ruolo di traduttore. È dall'edizione 1983 che egli figura invece come autore dell'opera; e il titolo non è più Evgenij Onegin, bensì Eugenio Onieghin di Puškin in versi italiani. Anche le note hanno un'evoluzione significativa in tal senso. Sull'«Almanacco dello Specchio», e anche nell'edizione 1975, esse sono a cura di Joanna Spendel e risultano ampie, limitrofe ai versi e ancora con molti riferimenti alla cultura russa. Nel corso delle edizioni tali note risultano invece ridotte ed emarginate, prima a fine capitolo (dal I983) e poi in forma di «sommarie indicazioni», ,2 a firma di Giudici stesso, che l'autore fornisce in sede di Nota preliminare (nel 1999).

Se nel peritesto avviene una scomparsa graduale del ruolo di Joanna Spendel, parallela a una forma di "appropriazione" dell'opera da parte del poeta, pubblicamente invece la slavista non prende mai parola. Ora, dopo un silenzio che perdurava dagli anni ' 70 , per la prima volta Spendel offre, sollecitata da una mia breve intervista, un racconto emozionato del sodalizio con Giudici. Queste le parole che usa per descrivere il lavoro di traduzione sull'Onegin:

si procedette nel lavoro nel modo seguente: ogni strofa veniva tradotta da me in prosa e accanto indicavo le molteplici varianti lessicali. Questo foglio veniva consegnato a Giudici che dava alla strofa l'impronta poetica. Spesso questo semplice processo veniva accompagnato da lunghissime conversazioni-discussioni al telefono e mentre io difendevo la filologia, Giudici reclamava la libertà dell'interpretazione poetica. Per Giudici è stato un lavoro di vari mesi prima di trovare il metro giusto, quello più assomigliante alla lingua originale, cioè alla tetrapodia giambica russa. La traduzione di Giudici tornava poi a me e io mi limitavo a segnalare l'allontanamento dal testo originale che Giudici non sempre accoglieva. In questa fase di traduzione Giudici aveva rinunciato all'abituale macchina da scrivere; ogni verso veniva trascritto e corretto a mano e solo nell'ultima stesura per l'editore era trascritto a macchina. ${ }^{13}$

commissione dottorale composta da P. De Marchi (supervisore), T. Crivelli, G. Ziffer, R. Zucco, F. Strologo, P. Benzoni, a.a. 2015/2016.

II Giovanni Giudici, Nota del traduttore, in Aleksandr Sergeevič Puškin, Evgenij Onegin (Eugenio Onieghin), traduzione in versi italiani di Giovanni Giudici, Milano, Garzanti, 1975, pp. XXIII-XXV.

I2 Giovanni Giudici, Eugenio Onieghin di Aleksandr S. Puškin in versi italiani, prefazione di Gianfranco Folena, Milano, Garzanti, I999, p. 2.

I3 Per l'intervista completa rimando alla mia tesi di dottorato: Cerneaz, L'Onegin di Giudici, cit. 
Il poeta, quindi, prima di lavorare a quel dossier dalle molte belle copie di cui parla nella citazione in apertura (fase dattiloscritta di cui purtroppo non mi risulta esserci attestazione) operava un primissimo avvicinamento al testo originale tramite una traduzione di servizio nata dal lavoro con Joanna Spendel. Un metodo simile a quello descritto per la traduzione dal cèco di Jiř̀i Orten, con la collaborazione di Vladimir Mikeš:

Mikeš leggeva il cèco, mi diceva il significato letterale in italiano e (dove necessario) mi specificava le varie ulteriori implicazioni di lingua poetica: rime, figure retoriche, ambiguità semantiche ecc. Io lo seguivo e andavo avanti passo passo, come procedendo in una fitta foresta, con gli occhi bendati e tenuto per mano. ${ }^{14}$

È proprio di tale fase di lavoro, antecedente a quella usuale davanti alla macchina a scrivere e fino ad ora sconosciuta, che ho a disposizione, grazie a Spendel, una prova inedita. Si tratta di una copia xerostatica, eseguita dalla stessa Spendel, delle pagine di un "quaderno" - secondo la sua definizione - interamente manoscritto da Giudici: sono I9 pagine A4, dove la riproduzione del "quaderno" occupa la parte in alto a destra del foglio, nella misura di $20 \times 13 \mathrm{~cm}$. Dalla riproduzione sembra che si tratti di un blocco-appunti con rilegatura in alto, su cui il poeta scrive solo sul recto con diverse penne a sfera, di colore non identificato. Tale testimone, che nomino QS, attesta il lavoro di Giudici su una parte del capitolo VI dell'opera (stanze VI-XXV; XXVIII-XLVI). Ogni pagina propone tendenzialmente la scrittura di due stanze, che sono alternativamente numerate in numero arabo o romano. In diversi casi la fotocopiatura taglia le parti superiore (probabilmente a causa della rilegatura) e laterale della pagina, non rendendo leggibili alcune porzioni del testo. Il manoscritto presenta una scrittura omogenea e tendenzialmente in pulito. Si tratta appunto di una traduzione quasi parola per parola del testo originale, che assume così una forma che potremmo definire "prosa in versi" («ogni strofa veniva tradotta da me in prosa», afferma Spendel nella descrizione del lavoro; così anche Giudici traduttore di Frost: "traducevo in prima stesura quasi come se traducessi in prosa»). ${ }^{15}$ Per la sua stessa natura, il testo non mostra un'attenzione rimica né metrica, benché sia interessante notare il restringimento materiale della linea di scrittura realizzata da Giudici nel passaggio dalla prosa di servizio ai versi della prima edizione del 1975 per Garzanti, che qui nomino Gi e che sarà tenuta in considerazione per uno sguardo comparativo-evolutivo. Lo scritto è ricco di annotazioni variantistiche alternative di scrittura immediata: Giudici

I4 Giudici, Da un'officina di traduzioni, cit., p.3I.

I5 Ibidem, p. 27. 
trascriveva una traduzione interlineare registrando le varianti, che erano frutto delle precisazioni sinonimiche o delle riflessioni semantiche nate dal sodalizio traduttorio. A testimonianza del carattere non tardivo con cui il poeta interviene sul testo, si veda la linea di scrittura dei vv. 6-7 dalla stanza XXI: ${ }^{16}$

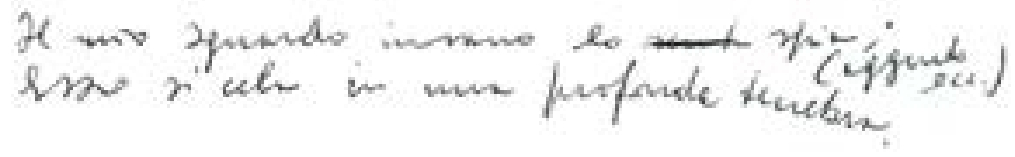

Il mio sguardo invano lo scruta spia \(agguato ecc.)〉, Esso si cela in una profonda tenebra.

L'inclinazione verso il basso del v. 7 è dettata certamente dal fatto che il rigo era già occupato dalla nota/inserzione. Le varianti, tendenzialmente, sono annotate dopo la conclusione del verso (sullo stesso rigo, leggermente distanziate) e sono appuntate entro parentesi quadre, o tonde, talvolta non chiuse; ma possono anche mancare di qualsiasi segnalazione grafica. In altri casi sono segnalate direttamente entro il testo - sempre tra quadre o tonde, anche non chiuse - subito dopo la lezione interessata o nei righi superiore o inferiore. Infine vi sono esempi di varianti annotate in spazi bianchi distanti dal luogo testuale cui fanno riferimento, anche senza segno di rimando.

A testimoniare la natura interlineare dello scritto, sono interessanti le registrazioni di alcune chiose esplicative, alle quali Giudici ricorre per fissare, quasi come fosse un promemoria, un'accezione semantica e l'atmosfera che intende ricreare nella sua proposta poetica. Restando sui versi appena ricordati, credo che il poeta appunti la nota "agguato" per rendere il significato del verbo del v. 6 («scruta», poi «spia»). Ma si veda ancora il primo verso della stanza XXIX:

Ecco già hanno scintillato (luccichio) le pistole

o il v. I2 della stanza XLV:

I6 Le trascrizioni che offro del manoscritto impiegano i segni di seguito descritti. Le barrette diagonali ( $|a b c|)$ indicano l'inserzione di una variante: gli interventi variantistici - qualsiasi sia la collocazione originaria - sono inseriti subito dopo la parola o le parole del verso a cui si riferiscono; in casi di riscrittura integrale di uno o più versi, sono invece trascritti a prosecuzione del verso/dei versi stessi. Le graffe corsive $(\{a b c\})$ segnalano integrazioni e note del curatore. Le parentesi tonde e quadre sono segni usati da Giudici per segnalare intervento variantistico immediato, che io trascrivo continuativamente, senza segno di inserzione. Per la trascrizione completa del quaderno rimando a Cerneaz, L'Onegin di Giudici, cit. 
Basta! Con l'anima serena \(limpida) chiara chiarezza \{prob.\}\}

dove Giudici annota, tramite l'introduzione di un sostantivo («luccichio», «chiarezza»), la connotazione che vuole evidenziare nell'uso del verbo, nel primo caso, e dell'aggettivo, nel secondo. Si guardi anche un'annotazione per la traduzione, alla stanza XXV:

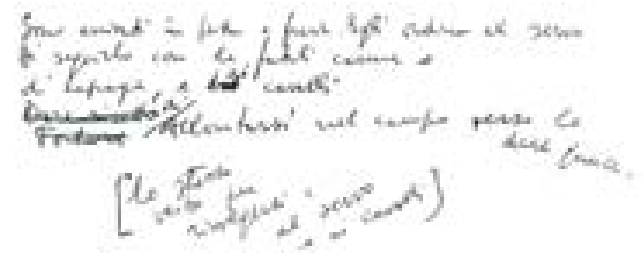

QS

Sono arrivati in fretta e furia. Egli ordina al servo

Di seguirlo con le fatali canne +

di Lepage, e dai ai cavalli

Dare ricetta $\backslash$ Portare $\backslash \backslash$ di $\backslash$ Allontanarsi nel campo verso le due querce.

Il poeta appunta una nota sintattico-grammaticale («[lo stesso verbo per rivolgersi e al servo e ai cavalli]»), che vuole rispettare nella sua versione.

È inoltre necessario soffermarsi sui casi in cui Giudici trascrive delle parole russe. Ne emerge anche una forma di incertezza, peraltro già evidenziata da parte della critica (soprattutto della russistica italiana). Si veda in merito il v. 5 della stanza XXXII, dove notiamo una trascrizione sbagliata di билось:

Il quel cuore erano (булось) ispirazione $\backslash[\operatorname{estro}\{\}\} \backslash$.

Giudici probabilmente scambia la "и" del verbo бйться (билось alla terza persona plurale) con "ы” del verbo essere быть - traduce infatti con «erano» e traslittera solo tale elemento differenziale con la "y" latina, ma mantenendo il resto del vocabolo in cirillico. Tale incertezza non è affatto occultata dal poeta, il quale affermava di «indovinare stentatamente i suoni corrispondenti alle lettere dell'alfabeto cirillico» ${ }^{17}$ Per Giudici la distanza dalla lingua è condizione di curiosità intellettuale e istanza ineludibile della poesia, che egli riconosce come «lingua straniera in grado ulteriore (o lingua strana, tout court)». ${ }^{18}$

È più interessante rilevare quanto, sin da questa fase di ricezione inter-

I7 Giudici, Per un Onieghin italiano, cit., p. 194.

I8 Giudici, Per passione e su commissione, cit., p. V. 
lineare dell'opera, sia già vivida la voce e la memoria poetica di Giudici. Si prenda ad esempio il v. I della stanza XXXI, dove il poeta inserisce la traslitterazione di «кладет», che probabilmente egli appunta per il futuro ripensamento (dall'edizione del '75) della costruzione di frase che è a cavallo con la stanza precedente, la XXX. Riporto gli ultimi due versi della stanza XXX e il primo della stanza XXXI dalla versione originale e nella trascrizione da QS e GI, separati da un interrigo bianco:

\author{
QS \\ L'ora fissata [fatale, del destino]: il poeta \\ Lascia cadere la pistola, senza una parola, \\ Al petto si porta (kladet) pian piano la mano \\ GI \\ L'ora è scoccata: non trattiene \\ L'arma il poeta, muto preme \\ Con la mano un poco il petto \\ Часы урочные: поэт \\ Роняет молча пистолет,
}

На грудь кладет тихонько руку

Notiamo che a partire da Gi l'azione del verbo класть («кладет», 'cadere in disperazione') passa dal primo verso della stanza XXXI dell'originale e della sua ricezione in QS all'ultimo verso della stanza XXX. Esso assume inoltre un'accezione diversa: parafrasando i versi, se in QS Lenskij "porta la mano al petto", da GI in poi "preme il petto con la mano". I versi rivelano una particolare attenzione stilistica: si noti la struttura chiastica che si sviluppa sui tre versi, mantenendo al centro il soggetto, ovvero il poeta, per una successione del tipo V/OSV/O. Il verbo premere inoltre, lontano dal suo oggetto e collocato solo alla fine del verso successivo, sembra alludere anche all'atto del premere il grilletto della pistola. Si noti anche come gli enjambements e la punteggiatura dettino una lettura sincopata, che il ricco ordito fonetico di $/ \mathrm{m} / \mathrm{e} \mathrm{di} / \mathrm{p} /$ asseconda: arma, poeta, Muto, preme, мano, poco, petto.

Anche al v. 3 della stanza XXXII Giudici segna in chiusura di verso la parola originale russa: si tratta di «лапоть», calzari popolari che il poeta dapprima traduce in «cioce», e poi, da GI, in «pianella» di manzoniana memoria. ${ }^{19}$ Un altro richiamo intertestuale, qui esplicito, è alla conclusione del

I9 «Lucia ... portava ... una corta gonnella di filaticcio di seta, a pieghe fitte e minute, due calze vermiglie, due pianelle, di seta anch'esse, a ricami», in Alessandro Manzoni, I promessi sposi 
v. 3 della stanza XXXVI, che riporto nell'interezza da QS:

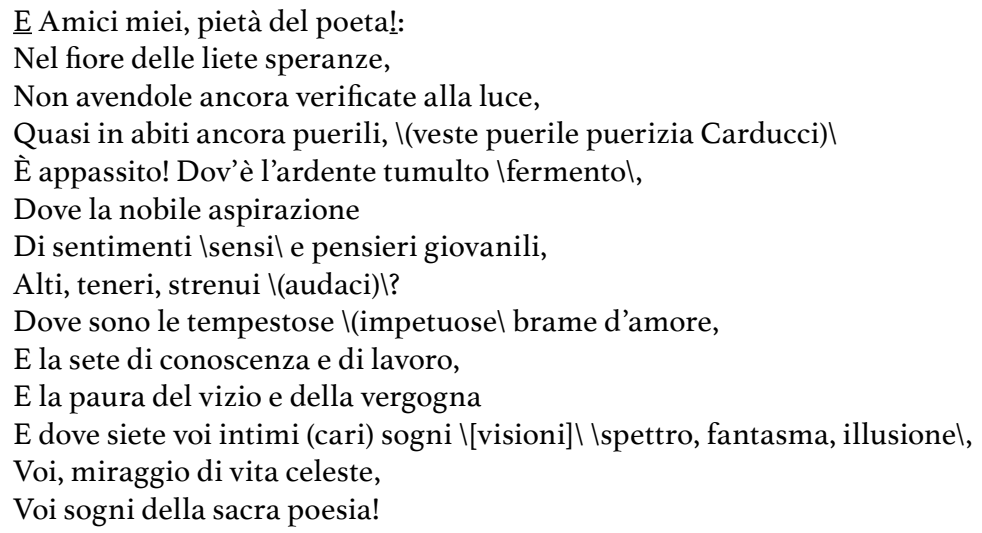

L'occorrenza di puerizia in Carducci è in Per la morte di Napoleone Eugenio, delle Odi Barbare: «e le morbide / chiome fiorenti di puerizia» (vv. 9-Io). Il riferimento floreale è presente anche nella stanza dell'Onegin, dove il fiore nella giovinezza è in pieno sboccio e colore («Liete speranze in pieno fiore», v. 3), fino all'appassimento con la morte («In vesti quasi ancor puerili / Appassì! [...]», vv. 4-5). ${ }^{20}$ Viene subito in mente anche Pianto antico, dove, come nell'Onegin, viene affrontato il tema della morte prematura. Qui la vita trova ancora una metafora floreale: «Lalbero a cui tendevi / La pargoletta mano, / Il verde melograno / Da' bei vermigli fior», vv. I-4; «tu fior della mia pianta», v. 9; «Estremo unico fior», v. I2. Ma è anche importante ravvisare che la $m u-$ tezza del giardino dove Carducci non sente più la voce del figlio («Nel muto orto solingo», v. 5) ricorre ripetutamente nella descrizione della morte di Lenskij, anche nelle altre stanze del capitolo: «L'arma il poeta, muto preme», (stanza XXX, v. I4); «Tutto è buio e silenzio adesso / Come una casa abbandonata; / Esso è muto per sempre. È sbarrata» (stanza XXXII, vv. 9-II); «E sordo e muto è diventato / $\mathrm{Al}$ vostro appello disperato?» (stanza XXXIV, vv. I3-I4).

Un aspetto importante che QS permette di approfondire è quello sulla genesi della rima. Genesi che risulta di particolare rilievo per l'Onegin: sappiamo infatti che la stanza di quattordici versi del romanzo è sempre organizzata in una successione fissa di sequenze rimiche alternate (AB'AB'), baciate (CCD'D'), incrociate (EF'F'E), con il sigillo di un distico (G'G'). Uno schema chiuso e rigido, che tuttavia Giudici sceglie come principio di organizzazione della stanza, pur ammettendo di non aver «potuto evitare il

[1827], a cura di Alberto Chiari e Fausto Ghisalberti, Milano, Mondadori, 1954, p. 38 (capitolo II).

20 Tutte le citazioni provengono dal testimone GI. 
ricorso con qualche frequenza a rime povere, rime false o anche semplici assonanze, a parte pochissimi casi di resa dichiarata all'inevitabilità della trasgressione». ${ }^{21}$ Un'attestazione esplicita dell'attenzione per la rima è data nella stanza XXV, dove ai vv. 5-6 Giudici segnala tramite una parentesi graffa, a cui affianca l'appunto «rima», due parole legate dalla stessa forma desinenziale, come in una sorta di appunto per la successiva sistemazione rimica del testo da GI:

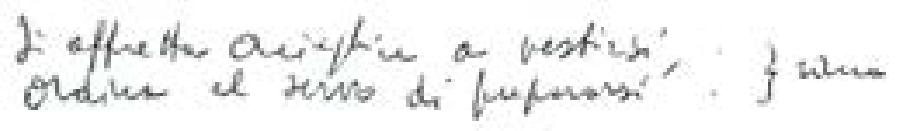

\author{
QS \\ Si affretta Onieghin a vestirsi \\ Ordina al servo di prepararsi \\ GI \\ Si affretta Onieghin a abbigliarsi, \\ Ordina al servo di apprestarsi
}

Ma il testimone fa soprattutto apprezzare quanto la stratificazione variantistica riveli una forte tensione rimica. Si guardi ai vv. 3-4 della stanza $\mathrm{XIV}$, dove troviamo anche un'ulteriore conferma della natura "letterale" dello scritto:

QS
«Perché ieri sera sei sparito così presto?»
Era la prima domanda di Olenka.
In Lienskij tutti i sentimenti fermarono,
E tacito chinò [tirò] il naso. \[si attristò]\} $\end{array}$

GI

«Perché iersera sei sparito

Così presto?» gli domandò.

E Lienskji tutto ammutolito

E confuso si rattristò.

«Зачем вечор так рано скрылись?»

2I Giudici, Per un Onieghin italiano, cit., pp. 198-I99. 
Был первый Оленькин вопрос.

Все чувства в Ленском помутились,

И молча он повесил нос.

In QS notiamo la riproduzione esatta delle parole del russo nell'immagine del chinare il naso, dove Puškin fa riferimento alla formula idiomatica повесить нос che ha l'accezione di 'cadere in disperazione'; ${ }^{22}$ usa inoltre la parola «naso» per riferirsi, pars pro toto, al volto di Lenskij, per una verosimile ragione rimica (вопрос : нос). Alla fine del rigo Giudici appunta il significato di tale abbassamento del volto, ovvero il rattristarsi di Lenskij, verbo che da GI inserisce al passato remoto («si rattristò»), così da trovare un richiamo al «domandò» del v. 2. Parallelamente, «sparito» del v. I viene spostato in clausola, per trovare un'eco nella confusione di Lenskij, che dapprima descritta come un blocco dei sentimenti («In Lienskij tutti i sentimenti fermarono») diviene poi una forma, ancora, di mutezza («E Lienskji tutto ammutolito»). Si vedano anche i vv. IO-I4 dalla stanza XXXVI:

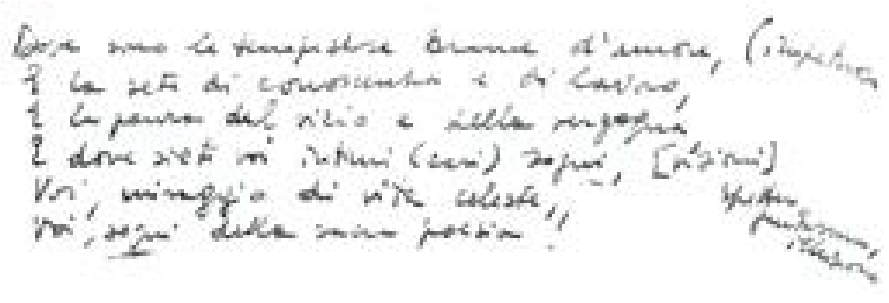

QS

Dove sono le tempestose \(impetuosel brame d'amore,

E la sete di conoscenza e di lavoro,

E la paura del vizio e della vergogna

E dove siete voi intimi (cari) sogni \[visioni] $\backslash \backslash$ spettro, fantasma, illusionel,

Voi, miraggio di vita celeste,

Voi sogni della sacra poesia!

che in GI diventano

Dove le brame d'amore inquiete,

La sete di scienza e lavoro,

La sua paura del disdoro?

Dove siete o visioni segrete.

22 Grande dizionario russo-italiano, a cura di Boris Majzel', Nadežda Skvorcova, terza edizione, Mosca, Russkij Yazyk, 200I. Anche su Kovalev si legge «чмо мы нос повѐсил? cos'hai da scoraggiarti tanto?», Dizionario russo italiano, italiano russo, a cura di Vladimir Kovalev, terza edizione, Milano, Zanichelli, 2007. 


\section{Del cielo indicanti la via, \\ Voi, sogni di sacra poesia!}

In QS le «brame d'amore» del v. Io sono «tempestose», poi, nella variante alternativa, «impetuose» e infine, da GI, «inquiete». Sempre in QS Giudici sottolinea i «sogni» dei vv. I2 e I4, probabilmente come appunto per evitare una futura ripetizione. $\mathrm{Al}$ v. I2 il poeta infatti annota una variante alternativa («visioni») arricchita da ulteriori varianti ancora non declinate nel numero («spettro, fantasma, illusione»). Da Gi egli opta per «visioni» che, non più «intime» o «care», diventano «segrete». Notiamo allora che la rima inquiete : segrete si radica proprio nella riflessione variantistica e nello spostamento in punta di verso della definizione aggettivale. Prendiamo ancora la prima quartina della stanza XLII:

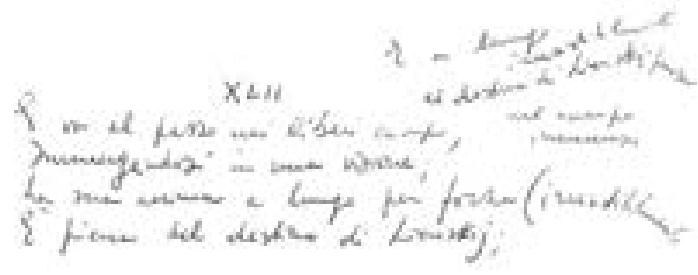

QS

E va al passo nei liberi campi \nel campo immensol,

Immergendosi in una visione;

La sua anima a lungo per forza \(irresistibilmentel

È piena del destino di Lienskij; $\backslash$ E a lungo irresistibilmente al destino di

Lienskij pensal

GI

Nel sogno assorta, lentamente

Va poi per la campagna immensa;

E a lungo e irresistibilmente

Al destino di Lienskij pensa

И шагом едет в чистом поле,

В мечтанья погрузясь, она;

Душа в ней долго поневоле

Судьбою Ленского полна;

Nella resa dell'avverbio russo «поневоле» del v. 3, Giudici prima registra in QS «per forza», che poi chiosa con la variante alternativa «irresistibilmente». Tale uscita avverbiale trova poi in GI un perfetto richiamo rimico, grazie alla trasformazione di «Al passo» del v. I («шагом») nell'avverbio «lenta- 
mente». Anche l'aggettivo «immenso» della variante del v. I di QS passa in Gi al v. 2 per richiamare in rima «pensa».

Gli esempi, non esaustivi, confermano la forte tensione rimica presente a questa altezza della costituzione testuale. Come ricorda Roberto Antonelli, «la rima è realmente un problema, il problema, della scrittura poetica rimico-ritmica», ${ }^{23}$ tanto che spesso «iniziare dalla fine è una necessità intrinseca». ${ }^{24}$ Si delinea così un piano genetico ma che definirei paradigmatico della scrittura dove la rima ha senza ombra di dubbio una funzione anteriore. Sarà soprattutto dall'edizione ' 83 che Giudici si muoverà anche verso soluzioni rimiche di grado zero, senza per questo ledere la funzione organizzativa della rima, che il poeta propone o disattende nell'antagonismo tra osservanza e inadempienza:

Ho bisogno di una regola fissa, anche per trasgredirla. Questo io lo chiamerei un effetto di catechismo. Ho bisogno di regole da rispettare, per poterle far fesse, to cheat them, come si dice in inglese. ${ }^{25}$

Il modello rimico rimarrà paradigmaticamente anteriore, ma, sul piano sintagmatico, secondario. Saranno altre le spinte stilistiche che Giudici decide di assecondare: quelle metrico-ritmiche e sintattiche.

sara.cerneaz@gmail.com

23 Roberto Antonelli, Tempo testuale e tempo rimico. Costruzione del testo e critica nella poesia rimata, «Critica del testo», I/I, I998, pp. I77-20I, p. I85.

24 Ibidem, p. I9I.

25 Ferdinando Camon, Giovanni Giudici, in Id., Il mestiere di poeta. Conversazioni critiche, Milano, Garzanti, 1982, pp. 15I-167, p. 154 . 